\title{
One-Dimensional Spin Glass
}

\author{
M. BŁAszyK And B. Fechner \\ Institute of Physics, A. Mickiewicz University \\ Umultowska 85, 61-614 Poznań, Poland
}

(Received November 21, 2005; revised version May 4, 2006)

\begin{abstract}
Thermodynamics of one-dimensional finite-ranged Ising model with quenched disorder introduced by random admixture of bonds with second neighbours by means of transfer-matrix method was determined. The influence of frustrations and competition on the internal energy, specific heat, correlations functions, and susceptibility was studied.
\end{abstract}

PACS numbers: 05.50.+q, 75.10.Nr, 75.50.Lk

\section{Introduction}

The physical understanding of infinite-ranged spin glasses began with the exact replica symmetry breaking solution of the Sherrington-Kirkpatrick model and the Thouless-Anderson-Palmer approach to the mean field theory [1]. Since that time we have a deeper appreciation of the mathematical problem that we are faced with dealing with quenched disorder. However, this does not apply to the finite-ranged spin glasses for which little exact results are known. In this case a number of one- or two-dimensional models comprising disorder and frustration have been investigated but exact results were obtained only for systems with some restrictions imposed on the frustration.

The ground state of one-dimensional systems with random field or random exchange integrals has been studied by Derrida et al. [2], Williams [3], and Doman [4]. Besides the strictly one-dimensional models Derrida et al. [2] investigated the ground state of a strip consisting of squares, imposing various conditions on the distribution of exchange integrals. Morita and Horiguchi [5] studied the ground state of a linear lattice composed of triangles which is topologically equivalent to a linear chain with the first and second neighbours interactions. The authors introduced three models. The first and the only one, which has been studied by these authors, assumes the bonds with the first neighbours to be ferromagnetic or antiferromagnetic and those with the second neighbours to be ferromagnetic. In other words, the nearest neighbour bonds are random while all the second neighbour bonds are the same. In the second model the first neighbours bonds are 
assumed to be the same in the whole lattice while the next nearest neighbours bonds are ferro- or antiferromagnetic at random. In the third model the bonds with both first and second neighbours are random. The ground states of the second and the third models have been studied by Fechner and Błaszyk [6].

For nonzero temperatures some properties of a one-dimensional model with competition between the exchange interaction and external field were investigated by Doman and Williams [7]. They found its free energy in two approximations. Once taking into account only the lowest excited states, i.e. for temperatures close to zero, and the second time assuming that the exchange integrals are periodically distributed throughout the lattice.

In this paper we discuss the temperature properties of a model which is close to the model II of Morita and Horiguchi but differs from it by the assumption that the random exchange integrals take on the values $J$ and 0 instead of $J$ and $-J$. This choice of the exchange integral preserves the randomness and frustration in the system, and at the same time enables exact calculation of the thermodynamical properties of the system at any temperature.

The aim to discuss such a model is twofold. Firstly, our task is to obtain some precise results concerning short-range, disordered and frustrated systems at any nonzero temperature for which, to the best of our knowledge, there are no available results. Secondly, the presented rigorous approach to the model can provide a testing ground for approximate methods used for analogous systems with quenched disorder.

\section{The model}

We consider a one-dimensional random Ising model described by the Hamiltonian

$$
\boldsymbol{H}=-J_{1} \sum_{i} \boldsymbol{S}_{i} \boldsymbol{S}_{i+1}-\sum_{i} I_{2, i} \boldsymbol{S}_{i} \boldsymbol{S}_{i+2},
$$

where $S_{i}= \pm 1$. The exchange integral $J_{1}$ between nearest neighbours, which may be ferro- or antiferromagnetic is assumed to be constant throughout the lattice while the exchange integral $I_{2, i}$ pertaining to the second neighbours is a stochastic quantity which takes on two values: $J_{2}$ with the probability $p$ and zero with the probability $1-p$. For $J_{1}>0, J_{2}<0$ and $\left|J_{2}\right|=J_{1}$ our model exhibits frustration. In this case it contains only frustrated plaquets, i.e. does not contain any closed loop enclosing an odd number of triangles with satisfied interactions. Thus it may be looked upon as a diluted fully frustrated model. Since the distribution of the frustrated plaquets is random, then our model holds both essential characteristics of a spin glass: frustration and disorder. Moreover it has the same ground state degeneracy for any $p$ as the second of the $\pm J$ model of Morita and Horiguchi which has been found in our previous paper [6]. This conclusion can be deduced from the fact that the triangles with $J_{2}=0$ in this model contribute to the ground state degeneracy in the same way as do the unfrustrated triangles in their models. 
Thus, one can expect that thermal properties of all these models in zero external field and at low temperatures will be similar.

\section{The free energy and the method of calculation}

The properties of one-dimensional Ising model without disorder are usually found by applying the transition matrix method [8]. In this case the unit cell consists of two nearest neighbour bonds and two next nearest neighbour bonds. Let us consider the problem that appears when disorder is introduced. In this case four transition matrices are needed. If the matrices commute, the thermodynamic properties of a system with a fixed sequence of the admixtured bonds depend entirely on their concentration. When the matrices do not commute, which is the case in our model, the physical properties also depend on the permutation of the matrices. For this reason we should proceed as follows. For each of the possible $4^{N / 2}$ sequences of exchange integrals, where $N$ is the number of sites, the product of transition matrices arranged in appropriate order should be multiplied by the probability of finding such a configuration. The sum of all such terms gives the free energy of the system. For large $N$, however, this procedure becomes arduous.

Introducing the pseudospins $\sigma_{i}=S_{i} S_{i+1}$ as the new variables, Hamiltonian (1) can be rewritten in the form

$$
\boldsymbol{H}=-J_{1} \sum_{i} \sigma_{i}-\sum_{i} I_{2, i} \sigma_{i} \sigma_{i+1} .
$$

This transformation does not change the number of the degrees of freedom of the system, so the partition function remains unchanged. The Hamiltonian (2) has the form of one-dimensional diluted system with the nearest neighbour interactions $I_{2, i}$, in an external field $J_{1}$. After the transformation the unit cell contains one lattice point and one bond only so the system can be described by two noncommuting $2 \times 2$ transition matrices. However, the main advantage of this transformation does not rely on the reduction of the unit cell and lowering thereby the dimension of the matrices but on the fact that the system considered, after the transformation, breaks up into clusters with the limits determined by the localization of the exchange integrals $J_{2}=0$ and this makes the clusters contributions to free energy additive

$$
F=\sum_{c} F_{c},
$$

where $F_{c}$ is the contribution to the free energy coming from the cluster $c$. The fact that the fragmentation into clusters is fully determined by the distribution of the exchange integrals and unlike the spin glasses does not depend on temperature is an important simplification of our model.

In order to find the free energy of one cluster we introduce the following transition matrices: $\boldsymbol{T}_{1}$ for the extreme left bond of the cluster, $\boldsymbol{T}$ for the bonds inside the cluster, and $\boldsymbol{T}_{N}$ for the extreme right bond of the cluster. They are given by 


$$
\begin{aligned}
& \boldsymbol{T}_{1}=\left[\begin{array}{ll}
\exp \left(K_{2}+3 K_{1} / 2\right) & \exp \left(-K_{2}+K_{1} / 2\right) \\
\exp \left(-K_{2}-K_{1} / 2\right) & \exp \left(K_{2}-3 K_{1} / 2\right)
\end{array}\right], \\
& \boldsymbol{T}_{N}=\left[\begin{array}{ll}
\exp \left(K_{2}+3 K_{1} / 2\right) & \exp \left(-K_{2}-K_{1} / 2\right) \\
\exp \left(-K_{2}+K_{1} / 2\right) & \exp \left(K_{2}-3 K_{1} / 2\right)
\end{array}\right], \\
& \boldsymbol{T}=\left[\begin{array}{ll}
\exp \left(K_{2}+K_{1}\right) & \exp \left(-K_{2}\right) \\
\exp \left(-K_{2}\right) & \exp \left(K_{2}-K_{1}\right)
\end{array}\right],
\end{aligned}
$$

where $K_{1}=\beta J_{1}, K_{2}=\beta J_{2}$, and $\beta=1 / k T$. The partition function for a cluster of $N$ bonds $(N \geq 2)$ can be expressed in the form

$$
Z_{N}=\sum_{S_{1}} \sum_{S_{N+1}}\left\langle S_{1}\left|\boldsymbol{T}_{1}\right| S_{2}\right\rangle\left\langle S_{2}\left|\boldsymbol{T}^{N-2}\right| S_{N}\right\rangle\left\langle S_{N}\left|\boldsymbol{T}_{N}\right| S_{N+1}\right\rangle
$$

In order to find $\boldsymbol{T}^{N-2}$ we first construct the diagonal matrix $\hat{\boldsymbol{T}}$ :

$$
\hat{\boldsymbol{T}}=\left[\begin{array}{ll}
\lambda_{+} & 0 \\
0 & \lambda_{-}
\end{array}\right],
$$

where $\lambda_{ \pm}=\exp \left(K_{2}\right) \cosh \left(K_{1}\right) \pm \sqrt{\exp \left(2 K_{2}\right) \sinh ^{2}\left(K_{1}\right)+\exp \left(-2 K_{2}\right)}$. The matrices $\boldsymbol{T}$ and $\hat{\boldsymbol{T}}$ are related by

$$
\hat{\boldsymbol{T}}=\boldsymbol{P}^{-1} \boldsymbol{T P}
$$

where $\boldsymbol{P}$ i $\boldsymbol{P}^{-1}$ have the following form:

$$
\begin{aligned}
& \boldsymbol{P}=\left[\begin{array}{cc}
1 & 1 \\
\exp \left(K_{2}\right)\left[\lambda_{+}-\exp \left(K_{2}+K_{1}\right)\right] & \exp \left(K_{2}\right)\left[\lambda_{-}-\exp \left(K_{2}+K_{1}\right)\right]
\end{array}\right], \\
& \boldsymbol{P}^{-1}=\frac{\exp \left(-K_{2}\right)}{\lambda_{+}-\lambda_{-}}\left[\begin{array}{cc}
\exp \left(K_{2}\right)\left[-\lambda_{-}+\exp \left(K_{2}+K_{1}\right)\right] & 1 \\
\exp \left(K_{2}\right)\left[\lambda_{+}-\exp \left(K_{2}+K_{1}\right)\right] & -1
\end{array}\right] .
\end{aligned}
$$

Thus, the partition function (5) can be expressed as

$$
Z=\sum \boldsymbol{T}_{1} \boldsymbol{P} \hat{\boldsymbol{T}}^{N-2} \boldsymbol{P}^{-1} \boldsymbol{T}_{N}=\lambda_{+}{ }^{N-2}(A+B)+\lambda_{-}{ }^{N-2}(A-B),
$$

where the summation runs over all matrix elements and

$$
\begin{aligned}
A= & \cosh \left(K_{1}\right) \exp \left(-2 K_{2}\right)+\exp \left(2 K_{2}\right) \cosh \left(3 K_{1}\right)+2 \cosh \left(K_{1}\right), \\
B= & {\left[2 \exp \left(-2 K_{2}\right) \cosh \left(2 K_{1}\right)+\exp \left(2 K_{2}\right) \sinh \left(3 K_{1}\right) \sinh \left(K_{1}\right)\right.} \\
& -\exp \left(-2 K_{2}\right) \sinh ^{2}\left(K_{1}\right)+2 \exp \left(-2 K_{2}\right) \cosh \left(2 K_{1}\right) \\
& \left.+2 \sinh ^{2}\left(K_{1}\right)\right]\left[\sinh ^{2}\left(K_{1}\right)+\exp \left(-4 K_{2}\right)\right]^{-1 / 2} .
\end{aligned}
$$

The probability of finding a given spin in a cluster with $N$ bonds, i.e. in a $N+1$ spin cluster, is equal to $(N+1)(1-p)^{2} p^{N}$. Mean free energy per spin in a cluster 
of $N$ bonds is $F_{N} /(N+1)$, so, in our case the free energy per spin takes the form

$$
F=\sum_{i=0}^{\infty} \frac{F_{i}}{i+1}(i+1)(1-p)^{2} p^{i}=(1-p)^{2} \sum_{i=0}^{\infty} F_{i} p^{i}
$$

The last expression can be rewritten as a sum of the five following components:

$$
\begin{aligned}
& f_{1}=-\frac{1}{\beta}(1-p)^{2} \ln \left[2 \cosh \left(K_{1}\right)\right] \\
& f_{2}=-\frac{1}{\beta}(1-p)^{2} p \ln \left[2 \exp \left(K_{2}\right) \cosh \left(2 K_{1}\right)+2 \exp \left(-K_{2}\right)\right] \\
& f_{3}=-\frac{1}{\beta} p^{3} \ln \left(\lambda_{+}\right) \\
& f_{4}=-\frac{1}{\beta}(1-p) p^{2} \ln (A+B), \\
& f_{5}=-\frac{1}{\beta} p^{2}(1-p)^{2} \sum_{N=0}^{\infty} p^{N} \ln \left[1+\left(\frac{\lambda_{-}}{\lambda_{+}}\right)^{N} \frac{A-B}{A+B}\right] .
\end{aligned}
$$

$f_{1}$ and $f_{2}$ are the contribution to the free energy coming from one- and two-spin clusters, and $f_{3}, f_{4}, f_{5}$ are the contributions from the clusters of $N \geq 2$ bonds.

\section{The internal energy and the specific heat}

The internal energy $E$ and specific heat $C$ can be found from $F$ and its temperature derivatives. Since the free energy is invariant under the transformation $J_{1} \rightarrow-J_{1}$ and its temperature derivatives preserve this symmetry, so do also $E$ and $C$.

When the interactions with the first neighbours are not competitive against those with the second neighbours, the energy is a monotonous function of temperature and concentration of the bonds with the second neighbours. When the competition arises, this dependence becomes more complex. Figure 1 illustrates the relation between the energy and the concentration of admixtured bonds, for different values of $J_{1}$ and $J_{2}$. When the interactions with first neighbours are twice as strong as those with second neighbours (curve $a$ in Fig. 1) the energy is a linear function of concentration and for a strictly one-dimensional case $(p=0)$ it is approximately equal to $J_{1}$. With increasing concentration of second neighbour bonds clusters of frustrated triangles appear and every frustrated triangle has an unsatisfied bond which contributes with higher energy. This unsatisfied bond may be either the one with a second neighbour and then one unsatisfied bond falls on one triangle, or the one with a first neighbour which then falls on a pair of triangles. Both these cases are equally energetically favourable and that is why the energy is a linear function of the bonds concentration. 


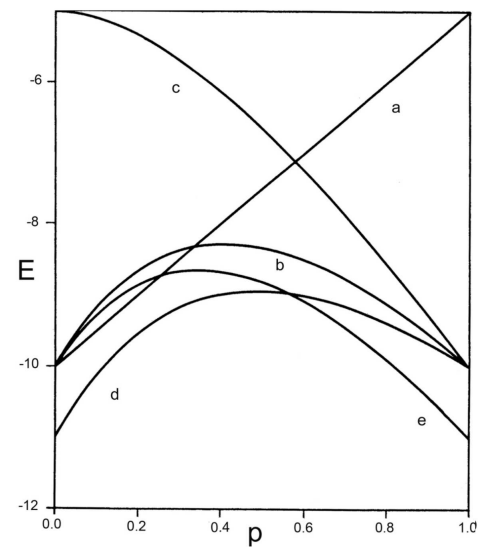

Fig. 1. The internal energy per site as a function of the concentration at the temperature $T=1$, for different values of exchange integrals. $a-J_{1}=20, J_{2}=-10, b-$ $J_{1}=10, J_{2}=-10, c-J_{1}=10, J_{2}=-20, d-J_{1}=11, J_{2}=-10, e-J_{1}=10$, $J_{2}=-11$.

The energy diagram for $\left|J_{1}\right|=\left|J_{2}\right|$ (curve $b$ in Fig. 1) looks quite different. In such a case it is much more energetically favourable to induce the unsatisfied bonds with the first neighbours. If the number of frustrated triangles in a cluster is odd, one of the triangles will have an excited bond which cannot simultaneously belong to its neighbouring frustrated triangles. The largest energy per spin is, of course, found for clusters composed of a single triangle, which occur with the probability $p(1-p)^{2}$. In consequence, the maximum of this function appears for $p=1 / 3$.

When $\left|J_{2} / J_{1}\right|=2$ (curve $c$ in Fig. 1), energetically more favourable are unsatisfied bonds with first neighbours and the relation between the energy and concentration is approximately linear. For small concentrations, $p \approx 0$, only separate triangles appear which do not change the energy with respect to the energy of the lattice without second neighbour bonds. This is why energy is only weakly dependent on $p$. Another extreme case occurs when $p \approx 1$. In this case the clusters of triangles join to form one large cluster. The intermediate cases illustrate the essential changes in energy when the ratio $\left|J_{1} / J_{2}\right|$ differs from 1.

Figure 2 shows the dependence of the specific heat $C / k$ on temperature $T=\beta^{-1}$ for the case when $J_{2}=J_{1}=1$, i.e. for a ferromagnetic model for different concentrations of second neighbours. Specific heat is an increasing function of admixtured bond concentration in the high-temperature region and a decreasing one in the low-temperature region. On the other hand, the maximum of the specific heat as a function of temperature decreases for small concentrations and increases for higher ones as $p$ grows larger and larger.

When the model is frustrated and $J_{2}=J_{1}=-1$, the specific heat, for all concentrations different from zero or one, tends to infinity when the temperature 


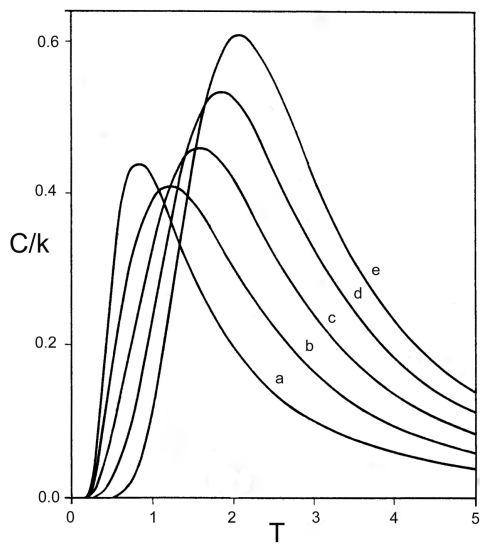

Fig. 2. Temperature dependence of the specific heat for $J_{1}=1, J_{2}=1$, and for the concentrations $p: a-0, b-0.25, c-0.5, d-0.75, e-1$.

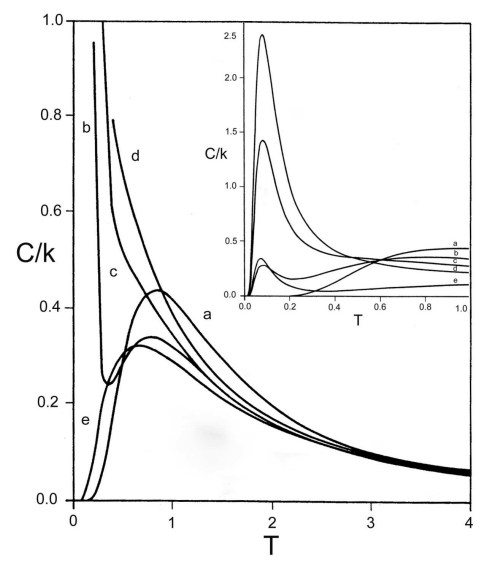

Fig. 3. Temperature dependence of the specific heat for $J_{1}=J_{2}=-1$ and for the same concentrations $p$ as in Fig. 2. The inset shows the specific heat in the case $J_{1}=1.1$ and $J_{2}=-0.5$.

tends to zero, Fig. 3. This behavior of specific heat is a consequence of the fact that the introduction of single bonds exponentially increases the degeneration with $N$. The behaviour of specific heat is the same when $\left|J_{2}\right|<J_{1}<2\left|J_{2}\right|$ which is related with the degeneracy of the ground state [6]. When $\left|J_{2}\right|<2\left|J_{1}\right|$, the specific heat for any $p$, tends to zero again (see the inset in Fig. 3). It is interesting to note that in this case the maximum of the specific heat occurs at temperatures lower than for the ferromagnetic case.

\section{Correlation functions}

We shall limit our considerations to the two-spin correlation functions only. In order to determine them we refer to the pseudospin notation $\sigma_{i}=S_{i} S_{i+1}$ intro- 
duced in Sect. 3. Any two-spin correlation function $\left\langle S_{i} S_{i+n}\right\rangle$ in this approach is equivalent to $\left\langle\sigma_{i} \sigma_{i+1} \ldots \sigma_{i+n-1}\right\rangle$. The above correlation function of pseudospins $\sigma$, further referred to as spins, depends on the position of the spins in the cluster and on their size. Therefore, similarly to the energy and specific heat, an interesting quantity is the correlation function averaged over the probability of finding a given sequence of clusters.

In order to determine the correlation function $\left\langle\left\langle S_{i} S_{i+1}\right\rangle\right\rangle_{J}=\left\langle\left\langle\sigma_{i}\right\rangle\right\rangle_{J}$ let us introduce the transition matrix $\boldsymbol{V}$ for two-spin cluster, the spin matrix $\boldsymbol{S}$ and its representation $\hat{\boldsymbol{S}}=\boldsymbol{P}^{-1} \boldsymbol{S P}$ :

$$
\begin{aligned}
& \boldsymbol{V}=\left[\begin{array}{cc}
\exp \left(K_{2}+K_{1}\right) & \exp \left(-K_{2}\right) \\
\exp \left(-K_{2}\right) & \exp \left(K_{2}-2 K_{1}\right)
\end{array}\right], \\
& \boldsymbol{S}=\left[\begin{array}{cc}
1 & 0 \\
0 & -1
\end{array}\right], \quad \hat{\boldsymbol{S}}=\left[\begin{array}{cc}
x & 1+x \\
1-x & -x
\end{array}\right],
\end{aligned}
$$

where

$$
x=\frac{\sinh \left(K_{1}\right)}{\sqrt{\sinh ^{2}\left(K_{1}\right)+\exp \left(-4 K_{2}\right)}} .
$$

Considering one-spin correlations we can distinguish the following cases:

1. Spin $\sigma_{i}$ belongs to a one-spin cluster. Then $\left\langle\sigma_{i}\right\rangle=\tanh K_{1}$ and as $1-p$ is the probability of finding a bond ending the cluster,

$$
A_{1}=(1-p) \tanh K_{1} \text {. }
$$

2. The contribution due to the spins ending the clusters can be written as

$$
B_{1}=\frac{\sum \boldsymbol{S} \boldsymbol{V}}{\sum \boldsymbol{V}}(1-p)+\sum_{i=0}^{\infty} \frac{\sum \boldsymbol{S} \boldsymbol{T}_{1} \boldsymbol{P} \hat{\boldsymbol{T}}^{i} \boldsymbol{P}^{-1} \boldsymbol{T}_{N}}{\sum \boldsymbol{T}_{1} \boldsymbol{P} \hat{\boldsymbol{T}}^{i} \boldsymbol{P}^{-1} \boldsymbol{T}_{N}} p^{i+2}(1-p) .
$$

The first and the second terms are the contributions due to two- and multispin clusters, respectively. The symbol $\sum$ stands for the sum of all matrix elements.

3. The spins from within the clusters bring the following contribution:

$$
C_{1}=\sum_{n=0}^{\infty} \sum_{i=0}^{n} \frac{\sum \boldsymbol{T}_{1} \boldsymbol{P} \hat{\boldsymbol{T}}^{i} \hat{\boldsymbol{S}} \hat{\boldsymbol{T}}^{n-i} \boldsymbol{P}^{-1} \boldsymbol{T}_{N}}{\sum \boldsymbol{T}_{1} \boldsymbol{P} \hat{\boldsymbol{T}}^{n} \boldsymbol{P}^{-1} \boldsymbol{T}_{N}} p^{n+2}(1-p) .
$$

Finally, the mean value of the spin, taking into account the probabilities of finding bonds starting the cluster, can be expressed as

$$
\left\langle\left\langle\sigma_{i}\right\rangle\right\rangle_{J}=\left(A_{1}+2 B_{1}+C_{1}\right)(1-p) .
$$

Similarly, to determine the mean value of two-spin correlation function $\left\langle\left\langle\sigma_{i} \sigma_{i+1}\right\rangle\right\rangle_{J}=\left\langle\left\langle S_{i} S_{i+2}\right\rangle\right\rangle_{J}$ we introduce the analogous expressions:

$$
A_{2}=\frac{\sum \boldsymbol{S} \boldsymbol{V} \boldsymbol{S}}{\sum \boldsymbol{V}} p(1-p)
$$




$$
\begin{aligned}
& B_{2}=\sum_{i=0}^{\infty} \frac{\sum \boldsymbol{S} \boldsymbol{T}_{1} \boldsymbol{S} \boldsymbol{P} \hat{\boldsymbol{T}}^{i} \boldsymbol{P}^{-1} \boldsymbol{T}_{N}}{\sum \boldsymbol{T}_{1} \boldsymbol{P} \hat{\boldsymbol{T}}^{i} \boldsymbol{P}^{-1} \boldsymbol{T}_{N}} p^{i+2}(1-p), \\
& C_{2}=\sum_{n=0}^{\infty} \sum_{i=0}^{n} \frac{\sum \boldsymbol{T}_{1} \boldsymbol{P} \hat{\boldsymbol{T}}^{i} \hat{\boldsymbol{S}} \hat{\boldsymbol{T}} \hat{\boldsymbol{S}} \hat{\boldsymbol{T}}^{n-i} \boldsymbol{P}^{-1} \boldsymbol{T}_{N}}{\sum \boldsymbol{T}_{1} \boldsymbol{P} \hat{\boldsymbol{T}}^{n+1} \boldsymbol{P}^{-1} \boldsymbol{T}_{N}} p^{n+3}(1-p) .
\end{aligned}
$$

Following the same procedure as that described above, one obtains for two-spin correlation function

$$
\left\langle\left\langle\sigma_{i} \sigma_{i+1}\right\rangle\right\rangle_{J}=\left(A_{2}+2 B_{2}+C_{2}\right)(1-p)+\left(A_{1}+B_{1}\right)^{2}(1-p) .
$$

The second term is a contribution brought by the spins $\sigma_{i}$ and $\sigma_{i+1}$ belonging to different clusters. In the derivation of Eq. (18) we made use of the fact that the correlation function of spins from different clusters is equal to the product of the correlations function of these spins. Following the same steps the three-spin correlation function can be written as

$$
\begin{aligned}
& \left\langle\left\langle\sigma_{i} \sigma_{i+1} \sigma_{i+2}\right\rangle\right\rangle_{J}=\left(A_{3}+2 B_{3}+C_{3}\right)(1-p)+\left(A_{2}+B_{2}\right)\left(A_{1}+B_{1}\right)(1-p) \\
& \quad+\left(A_{1}+B_{1}\right)^{2} A_{1}(1-p),
\end{aligned}
$$

where

$$
\begin{aligned}
A_{3} & =\frac{\sum \boldsymbol{S} \boldsymbol{T}_{1} \boldsymbol{S T}_{N} \boldsymbol{S}}{\sum \boldsymbol{T}_{1} \boldsymbol{T}_{N}} p^{2}(1-p), \\
B_{3} & =\sum_{i=0}^{\infty} \frac{\sum \boldsymbol{S} \boldsymbol{T}_{1} \boldsymbol{P} \hat{\boldsymbol{S}} \hat{\boldsymbol{T}} \hat{\boldsymbol{S}} \boldsymbol{P}^{-1} \boldsymbol{T}_{N}}{\sum \boldsymbol{T}_{1} \boldsymbol{P} \hat{\boldsymbol{T}}^{i} \boldsymbol{P}^{-1} \boldsymbol{T}_{N}} p^{i+2}(1-p), \\
C_{3} & =\sum_{n=0}^{\infty} \sum_{i=0}^{n} \frac{\sum \boldsymbol{T}_{1} \boldsymbol{P} \hat{\boldsymbol{T}}^{i} \hat{\boldsymbol{S}} \hat{\boldsymbol{T}} \hat{\boldsymbol{S}} \hat{\boldsymbol{T}} \hat{\boldsymbol{S}} \hat{\boldsymbol{T}}^{n-i} \boldsymbol{P}^{-1} \boldsymbol{T}_{N}}{\sum \boldsymbol{T}_{1} \boldsymbol{P} \hat{\boldsymbol{T}}^{n+2} \boldsymbol{P}^{-1} \boldsymbol{T}_{N}}(1-p) .
\end{aligned}
$$

Applying the same procedure we found expressions for the correlation functions $\left\langle\left\langle S_{i} S_{i+1}\right\rangle\right\rangle_{J}$ up to $n=20$.

The numerically calculated dependence of the correlation function $\Gamma$ on the distance $n$, at a fixed temperature and concentration of second neighbour bonds, is shown in Fig. 4. It is evident from these figures that in the case when frustration occurs $\left(J_{2}=-1\right)$ the correlations diminish faster than in the ferromagnetic $\left(J_{2}=1, J_{1}=1\right)$ or antiferromagnetic $\left(J_{2}=1, J_{1}=-1\right)$ cases. This is so because in the nonfrustrated case the bonds with second neighbours induce order whereas in the frustrated case they destroy it. In the ferromagnetic case the correlations diminish monotonously while in the antiferromagnetic case they oscillate changing the sign alternately. For both these systems a general character of the correlation function does not depend on temperature, coupling constant or concentration of nonzero bonds. If the system is frustrated, the periodicity of the correlation function depends on external parameters. 


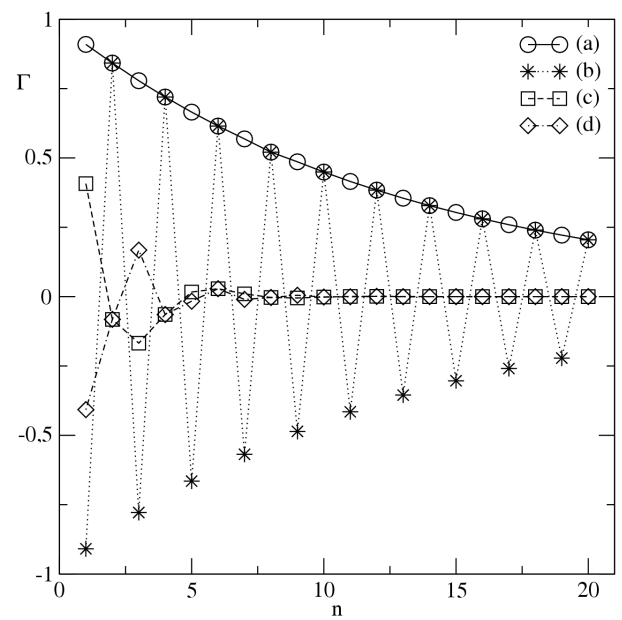

Fig. 4. The correlation function $\Gamma$ versus the spin distance $n$, for $T=1, p=0.5$ and for various first and second neighbours interactions: (a) $J_{1}=1, J_{2}=1$, (b) $J_{1}=-1, J_{2}=1$, (c) $J_{1}=1, J_{2}=-1$, (d) $J_{1}=-1, J_{2}=-1$. The lines are added as a guide for the eye.

\section{Magnetic susceptibility}

The magnetic susceptibility per site can be determined from the expression $\chi=\frac{\beta}{N}\left\langle\left\langle\left(\sum_{i} S_{i}\right)\left(\sum_{j} S_{j}\right)\right\rangle\right\rangle_{J}$,

which may be rewritten in the form

$$
\chi=\beta\left(1+2\left\langle\left\langle S_{i} S_{i+1}\right\rangle\right\rangle_{J}+2\left\langle\left\langle S_{i} S_{i+2}\right\rangle\right\rangle_{J}+2\left\langle\left\langle S_{i} S_{i+3}\right\rangle\right\rangle_{J}+\ldots\right)
$$

or, in the pseudospin approach,

$$
\chi=\beta\left(1+2\left\langle\left\langle\sigma_{i}\right\rangle\right\rangle_{J}+2\left\langle\left\langle\sigma_{i} \sigma_{i+1}\right\rangle\right\rangle_{J}+2\left\langle\left\langle\sigma_{i} \sigma_{i+1} \sigma_{i+2}\right\rangle\right\rangle_{J}+\ldots\right) .
$$

One could think that since the correlations decrease with distance, then using the above calculated 20 correlation functions, a satisfying approximate expression for the susceptibility could be obtained. It turns out, however, that the right hand side of Eq. (23) is a slowly convergent series and the final result in the low temperature region strongly depends on the number of the summed up terms.

The series (23) gives an exact expression for the susceptibility in the sense that its consecutive terms can be calculated exactly and only for technical reasons their number is limited to some $n$; in our case to $n=20$. However, if we assume that the size of the clusters does not exceed a given number of spins, then some correlation functions for any $n$ can be found and the infinite series of them summed up giving an expression for $\chi$ in a formally closed form.

In order to find multispin correlation function for any $n$ we shall at first determine the mean value of this function when all spins belong to the same cluster. In the case of one-, two- and three-spin clusters they are given by Eqs. (13), (17), and (20), respectively. In general, for $i>2$ we have 


$$
A_{i}=\frac{\sum \boldsymbol{S} \boldsymbol{T}_{1} \boldsymbol{P} \hat{\boldsymbol{S}}(\hat{\boldsymbol{T}} \hat{\boldsymbol{S}})^{i-2} \boldsymbol{P}^{-1} \boldsymbol{T}_{N} \boldsymbol{S}}{\sum \boldsymbol{T}_{1} \boldsymbol{T}^{i-2} \boldsymbol{T}_{N}} p^{i}(1-p) .
$$

As previously, we do not take into account the probability of finding a bond starting a cluster. Denoting by $S_{1}$ the sum over all such correlations we have

$$
S_{1}=\sum_{i=0}^{\infty} \frac{\sum \boldsymbol{S} \boldsymbol{T}_{1} \boldsymbol{P} \hat{\boldsymbol{S}}(\hat{\boldsymbol{T}} \hat{\boldsymbol{S}})^{i} \boldsymbol{P}^{-1} \boldsymbol{T}_{N} \boldsymbol{S}}{\sum \boldsymbol{T}_{1} \boldsymbol{T}^{i} \boldsymbol{T}_{N}} p^{i+2}(1-p)+A_{1}+A_{2} .
$$

Since, for each $p \neq 1$, the absolute value of $S_{1}$ is lower than 1 , the sum over such correlations which begin at the outset of a cluster and finish at the end of the same or another cluster is $S_{1} /\left(1-S_{1}\right)$. In the same way we find the sum of the correlation functions which concern only the inside spins of the cluster except for the first and the last ones

$$
S_{2}=\sum_{j=0}^{\infty} \sum_{n=0}^{\infty} \sum_{i=0}^{\infty} \frac{\sum \boldsymbol{T}_{1} \boldsymbol{P} \boldsymbol{T}^{i} \hat{\boldsymbol{S}}(\hat{\boldsymbol{T}} \hat{\boldsymbol{S}})^{j} \hat{\boldsymbol{T}}^{n-j} \boldsymbol{P}^{-1} \boldsymbol{T}_{N}}{\sum \boldsymbol{T}_{1} \boldsymbol{T}^{n+j} \boldsymbol{T}_{N}} p^{j+n+2}(1-p)
$$

The first three terms of the sum, for $j=0,1,2$ are the previously determined terms $C_{1}, C_{2}$, and $C_{3}$, respectively, expressed by Eqs. (15), (17), and (20). The sum of all correlations among the spins of which only one begins or ends the cluster can be written as

$$
\begin{aligned}
S_{3}= & \sum_{j=0}^{\infty} \sum_{i=0}^{\infty} \frac{\sum \boldsymbol{S} \boldsymbol{T}_{1} \boldsymbol{P} \hat{\boldsymbol{S}}(\hat{\boldsymbol{T}} \hat{\boldsymbol{S}})^{j} \hat{\boldsymbol{T}}^{i} \boldsymbol{P}^{-1} \boldsymbol{T}_{N}}{\sum \boldsymbol{T}_{1} \boldsymbol{T}^{j+i} \boldsymbol{T}_{N}} p^{i+j+2}(1-p) \\
& +\frac{\sum \boldsymbol{S} \boldsymbol{V}}{\sum \boldsymbol{V}} p(1-p) .
\end{aligned}
$$

The first three terms of this sum, over $j$, are equal to $B_{1}, B_{2}$, and $B_{3}$, respectively. Now, Eq. (23) can be rewritten in the form

$\chi=\beta\left\{1+2\left[S_{2}(1-p)+\left(S_{3}+1\right)^{2} \frac{S_{1}}{1-S_{1}}(1-p)+\left(S_{2}^{2}+2 S_{2}\right)(1-p)\right]\right\}$,

where the first term in the square bracket is the sum of all correlations within a single cluster without the spins beginning and ending this cluster, the second term is the sum of the correlations among all spins of at least one cluster, and the third one is the sum of the correlations among the spins in a single cluster including the spin ending this cluster or among the spins ending two different clusters.

In deriving (28) we have not specified the size of the clusters taken into account. It enters this expression only through the terms $S_{i}(i=1,2,3)$. Because this size can be chosen arbitrarily large, the right hand side of (28) represents the susceptibility exactly. The difference between (28) and (23) is that the former affords another kind of approximation to be done. Fixing the largest admissible cluster we are able to calculate all correlation functions not only up to $n$ coinciding with the size of the cluster but also for any sequence of adjacent clusters whose size does not exceed the assumed one.

Figures 5 and 6 show the temperature dependence of the susceptibility for a few concentrations $p$, and for different signs of the exchange integrals $J_{1}$ and $J_{2}$. 


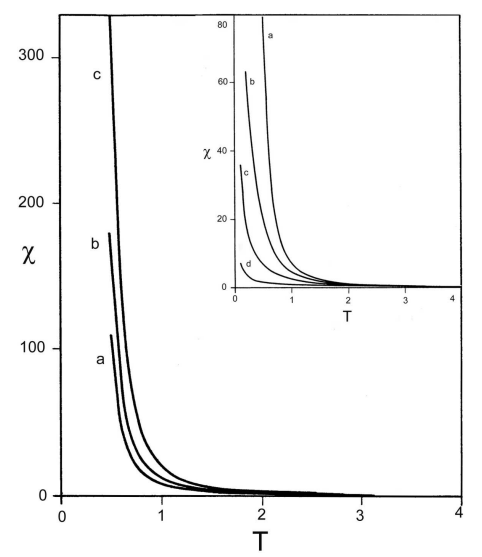

Fig. 5. Temperature dependence of the susceptibility for $J_{1}=1$ and $J_{2}=1$, for the concentrations $p: a-0.01, b-0.25, c-0.5$. The inset shows the susceptibility for $J_{1}=1$ and $J_{2}=-1$, for the concentrations $p$ equal to: $a-0.01, b-0.1, c-0.25$, $d-0.5$.

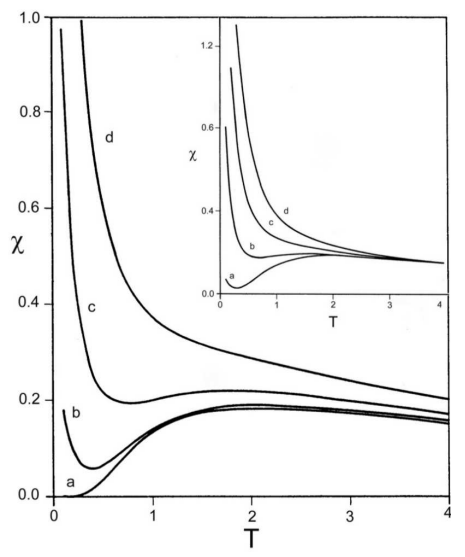

Fig. 6. Temperature dependence of the susceptibility for $J_{1}=-1$ and $J_{2}=1$, for the concentrations $p$ equal to: $a-0.01, b-0.1, c-0.25, d-0.5$. The inset shows the susceptibility in the case $J_{1}=-1$ and $J_{2}=-1$ for the same concentrations.

We report here only the results for $p \leq 1 / 2$ since the calculation of the susceptibility for higher $p$ values requires too much computer time. When the exchange integrals are ferromagnetic, Fig. 5, the susceptibility is a monotonously decreasing function of temperature, and the difference between the two figures comes from the fact that in the first case ferromagnetic and in the second one antiferromagnetic bonds with second neighbour are added at random to the ferromagnetic chain. An increase in the concentration of ferromagnetic bonds results in an increase in the susceptibility whereas increasing concentration of the antiferromagnetic bonds diminishes the susceptibility due to the appearance of some staggered spin ar- 
rangement. The diagrams shown in Fig. 6 that refer the case of antiferromagnetic bonds with the first neighbours have quite a different character. Admixed bonds with the second neighbours destroy the magnetic periodicity of the lattice which leads to an increase in the susceptibility, particularly well revealed at low temperatures. However, further addition of admixed bonds can be supposed to restore the periodicity and this would be the reason why the susceptibility decreases.

\section{Conclusions}

A comprehensive study was performed of the Ising model with the interactions with the first neighbours and random interactions with the second neighbours. The internal energy, specific heat, correlation functions, and susceptibility were determined. The system's energy behaviour as a function of concentration was explained in terms of frustration. The behaviour of the specific heat depends on the sign of $J_{2}$. When $J_{2}>0$, the specific heat maximum occurs at a temperature much higher than that of the maximum for $J_{2}<0$. The specific heat is particularly high when the ground state is degenerated, that is for $J_{2}<0$ and $\left|J_{2}\right|<J_{1}<2\left|J_{2}\right|$.

The system considered is below the lower critical dimension of the spin glass, so the appearance of this phase is not expected. The short-range ordering also exists in the paramagnetic phase and it can be the subject of analysis. For real spin glasses the specific heat maximum is decreased, a little broadened and shifted to a lower temperature relative to that of the ordered systems, hence it is reasonable to suppose that the mechanism responsible for a decrease in the temperature of the specific heat maximum is the competition of interactions.

Finally, let us point out the essential difference between our results and these obtained by the mean field method, which is the most frequently used approach to complicated systems. In our exact calculations the interaction energy within the cluster depends not only on its size but also on the position of each bond considered i.e. on their distribution in the cluster, while in the mean field approximation the interaction energy is determined by the nearest neighbours exchange interaction and an effective field that smooths out some details of the bonds at a greater distance away. Just for this reason we were able to get some insight into the effect of frustration and show how it influences the temperature dependence of the energy, the specific heat, the correlation function and the magnetic susceptibility by regulating the concentration of the bonds and their signs.

Looking for a theory which is methodologically nearest to our approach, the perturbation series expansions of the thermodynamic functions come to mind. This is especially true with reference to the high and low temperature series expansions of the Ising model that proved useful in testing the validity of closed form approximations [9]. These series expansions provide little understanding of the related physical processes, however, thanks to the exact calculations of a number of subsequent terms of the series, enable precise and reliable estimates of a variety of physical properties of the model they have been applied to. 
In our calculations a perturbation parameter does not appear. Instead, we build up a sequence of models of increasing size, each of which is calculated exactly. In this way on the one hand we get a rather slowly converging series of values of a given thermodynamic quantity, while on the other hand, however, we obtain some insight into the way the disorder and frustration control the thermodynamic behaviour of the system in the whole range of temperatures.

\section{References}

[1] M. Mezard, G. Parisi, M.M. Virasoro, Spin Glass Theory and Beyond, World Scientific, Singapore 1987, Chs. I, II, III.

[2] B. Derrida, J. Vannimenus, Y. Pomeau, J. Phys. C, Solid State Phys. 11, 4749 (1978).

[3] J.K. Williams, J. Phys. C, Solid State Phys. 14, 4095 (1981).

[4] B.G.S. Doman, J. Phys. C, Solid State Phys. 15, 5641 (1982).

[5] T. Morita, T. Horiguchi, J. Phys. C, Solid State Phys. 13, 6483 (1980).

[6] B. Fechner, M. Błaszyk, J. Phys. C, Solid State Phys. 19, 2785 (1986).

[7] B.G.S. Doman, J.K. Williams, J. Phys. C, Solid State Phys. 15, 1693 (1982).

[8] L.E. Reichl, A Modern Course in Statistical Physics, Edward Arnold Ltd, University of Texas Press, Austin 1980, p. 163.

[9] C. Domb, in: Phase Transitions and Critical Phenomena, Eds. C. Domb, M.S. Greene, Vol. 1, Academic Press, London 1974, p. 357. 